\title{
CMOS Voltage Reference Based on the Threshold Voltage of a MOSFET P-5-4
}

\author{
Ken Ueno, Tetsuya Hirose, Tetsuya Asai, and Yoshihito Amemiya \\ Department of Electrical Engineering, Hokkaido University, \\ Kita 14, Nishi 9, Kita-ku, Sapporo, Hokkaido, 060-0814, Japan \\ Phone: +81-11-706-7147, Fax: +81-11-706-7890, E-mail: k_ueno@sapiens-ei.eng.hokudai.ac.jp
}

\section{INTRODUCTION}

One of the promising areas of research in microelectronics is the development of ultra-low power analog LSIs that operate in the subthreshold region of MOSFETs [1]. To step toward such LSIs, we first need to develop a low-power voltage reference circuit that can operate with a very small current, several hundreds of nanoamperes or lower. However, with conventional circuitry, such reference circuits need a high resistance of several hundred megaohms, and therefore, they are difficult to implement in a small area on a chip. To solve this problem, we propose a novel voltage reference circuit without resistors. Our circuit combines the positive temperature coefficient of the thermal voltage with the negative temperature coefficient of a MOSFET threshold voltage to achieve a voltage reference with a zero temperature coefficient. The following sections describe this circuit in detail.

\section{Circuit Configuration}

\section{A. Operation Principle}

The voltage reference circuit we propose consists of a current source subcircuit and a bias-voltage subcircuit. The latter supplies a bias voltage to the former. Figure 1 shows the voltage reference circuits we proposed. The current source subcircuit is based on the basic $\beta$-multiplier self biasing circuit and uses a MOS resistor $\mathrm{M}_{\mathrm{R} 1}$ instead of an ordinary passive resistor. The bias-voltage subcircuit accepts the current through pMOS current mirrors and generates the reference voltage. The bias-voltage subcircuit is based on the translinear principle and uses a MOS resistor $M_{R 2}$. We operate all MOSFETs in the subthreshold region except for MOS resistors $\left(M_{R 1}\right.$ and $\left.M_{R 2}\right)$ in the strong-inversion and deep triode region. The details on the circuit operation are as follows.

The subthreshold MOS current $I_{D}$ can be expressed as

$$
I_{D}=K I_{0} \exp \left(\frac{V_{G S}-V_{T H}}{\eta V_{T}}\right)
$$

where $K$ is the aspect ratio $(=W / L)$ of transistors, $I_{0}(=\beta(\eta-$ 1) $\left.V_{T}^{2}\right)$ is the process-dependent parameter, $V_{T}\left(=k_{B} T / q\right)$ is the thermal voltage, $k_{B}$ is the Boltzmann constant, $T$ is the absolute temperature, $q$ is the elementary charge, $V_{T H}$ is the threshold voltage of a MOSFET, and $\eta$ is the subthreshold slope factor [2].

In the circuit in Fig.1, the current flowing in the circuit $I_{P}$ is determined by the ratio of $\mathrm{M}_{1}$ and $\mathrm{M}_{2}$ and the resistance of MOS resistor $M_{R 1}$, and is given by

$$
I_{P}=\beta\left(V_{R E F}-V_{T H}\right) \eta V_{T} \ln \left(\frac{K_{2}}{K_{1}}\right),
$$

where $\beta$ is the current gain factor. In the bias-voltage subcircuit, gate-source voltages of transistors $\left(V_{G S 3}\right.$ through $\left.V_{G S 7}\right)$

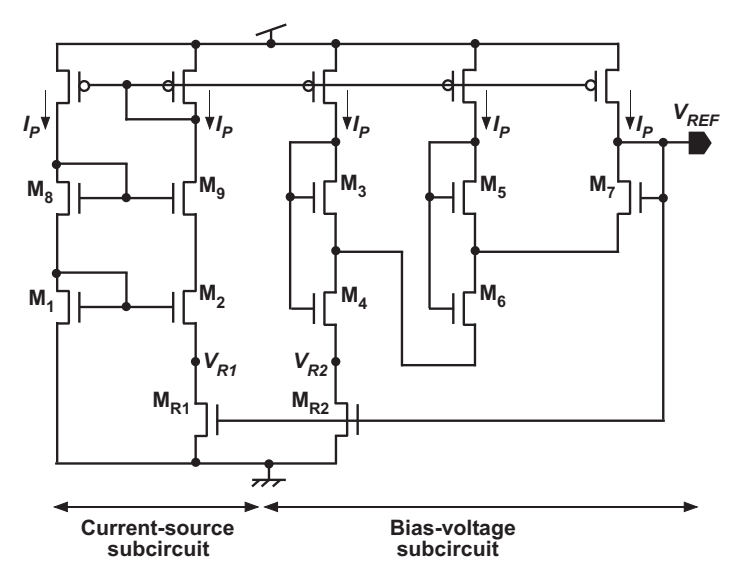

Fig. 1. Schematic of the proposed threshold voltage reference circuit.

and drain-source voltage of MOS resistor $\left(V_{R 2}\right)$ form a closed loop with the reference voltage $V_{R E F}$, so we find that

$$
\begin{aligned}
V_{R E F} & =V_{R 2}+V_{G S 4}-V_{G S 3}+V_{G S 6}-V_{G S 5}+V_{G S 7} \\
& =V_{G S 4}+\eta V_{T} \ln \left(\frac{2 K_{2}^{3} K_{3} K_{5}}{K_{1}^{3} K_{6} K_{7}}\right) .
\end{aligned}
$$

The reference voltage can be expressed by the sum of gatesource voltage $V_{G S 4}\left(=V_{T H}+\eta V_{T} \ln \left(I_{P} / K_{4} I_{0}\right)\right)$ and thermal voltage $V_{T}$ scaled by the transistor sizes. Because the gatesource voltage and the scaled thermal voltage have negative and positive temperature dependence, respectively, we can obtain a constant voltage with little temperature dependence, adjusting the size of transistors. Note that the threshold voltages and the current gain factors of source-coupled transistor pairs $\left(M_{3}-M_{6}, M_{5}-M_{7}\right)$ and MOS resistor pair $\left(M_{R 1}-M_{R 2}\right)$ can be canceled each other by this circuit configuration.

\section{B. Temperature Dependence}

The temperature dependence of the threshold voltage can be given by

$$
V_{T H}=V_{T H 0}-\kappa T,
$$

where $V_{T H 0}$ is the threshold voltage at absolute zero, and $\kappa$ is the temperature coefficient of the threshold voltage. On the condition where the voltage difference between reference voltage $V_{R E F}$ and threshold voltage at absolute zero $V_{T H O}$ is smaller than $\kappa T$, that is

$$
V_{R E F}-V_{T H 0} \ll \kappa T,
$$

temperature coefficient of reference voltage $V_{R E F}$ in Eq.(3) is given by

$$
\frac{d V_{R E F}}{d T}=-\kappa+\frac{\eta k_{B}}{q} \ln \left\{\frac{6 q \eta \kappa}{k_{B}(\eta-1)} \frac{K_{\beta} K_{2}^{3} K_{3} K_{5}}{K_{1}^{3} K_{4} K_{6} K_{7}} \ln \left(\frac{K_{2}}{K_{1}}\right)\right\} .
$$

We can obtain a constant voltage with a zero temperature coefficient by setting the aspect ratios such that $d V_{R E F} / d T=$ 


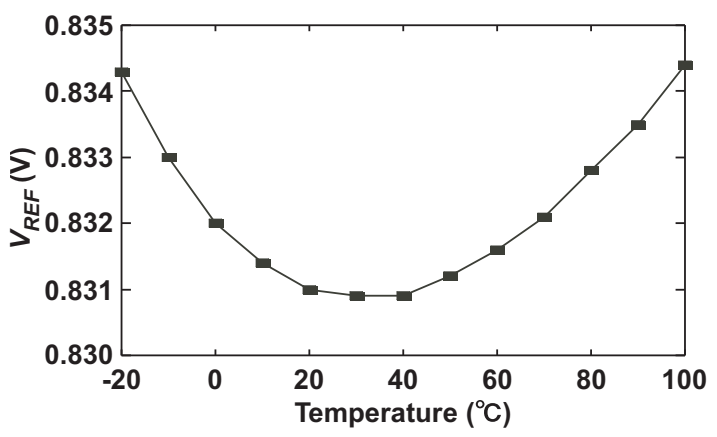

Fig. 2. Simulated output voltage as a function of temperature.

0 in Eq.(6). From Eqs.(2) - (6), the output voltage $V_{R E F}$ is given by

$$
V_{R E F}=V_{T H 0}+\eta V_{T} \ln \left(1+\frac{V_{R E F}-V_{T H 0}}{\kappa T}\right)=V_{T H 0} .
$$

Therefore, the circuit generates the threshold voltage of MOSFET at absolute zero temperature.

\section{RESUlts}

We confirmed the operation of the circuit by a SPICE simulation with a set of $0.35-\mu \mathrm{m} 2 \mathrm{P} 4 \mathrm{M}$ standard CMOS parameters and a $1.5-\mathrm{V}$ power supply. Figure 2 shows output voltage $V_{R E F}$ as a function of temperature from $-20{ }^{\circ} \mathrm{C}$ to 100 ${ }^{\circ} \mathrm{C}$. An almost constant output voltage can be obtained and average voltage was about $830 \mathrm{mV}$. The error in the output voltage was within $\pm 0.3 \%$ in the temperature range.

Figure 3 shows average output voltage $\overline{V_{R E F}}$ from $1 \mathrm{k}$ point Monte Carlo simulations assuming process spread and device mismatch in all MOSFETs. Because output voltage $V_{R E F}$ monitors the threshold voltage of nMOSFET, average output voltage $\overline{V_{R E F}}$ changed with process variation as shown in Fig.3-(a). Figure 3-(b) shows average output voltage $\overline{V_{R E F}}$ as a function of the threshold voltage $V_{T H}$. The output voltage had a linear dependence with threshold voltage. On the contrary, the errors of the output voltage with temperature were quite small and were within $\pm 0.3 \%$ as shown in Fig.4. This is because the temperature coefficient of the threshold voltage has little dependence on process variations. The temperature coefficient of the threshold voltage is expressed as

$$
\frac{d V_{T H}}{d T}=-(2 \eta-1) \frac{k_{B}}{q}\left\{\ln \left(\frac{\sqrt{N_{c} N_{v}}}{N_{a}}\right)+\frac{3}{2}\right\}+\frac{\eta-1}{q} \frac{d E_{g}}{d T},
$$

where $N_{a}$ is the channel doping concentration, $N_{c}$ and $N_{v}$ are the effective densities of states in the conduction and valence bands, respectively, and $E_{g}$ is the bandgap energy of silicon [2]. While the threshold voltage of a MOSFET changes significantly with the number of $N_{a}$, the temperature coefficient of the threshold voltage changes little because $N_{a}$ is contained in a logarithmic function.

Table I summarizes the performance of proposed circuits. The simulations showed the maximum power consumption, $1.2 \mu \mathrm{W}$ at $100{ }^{\circ} \mathrm{C}$. The change of the output voltage with the supply voltage $V_{D D}$ was within $\pm 0.5 \%$ in the range of 1.2 $\mathrm{V}$ to $3 \mathrm{~V}$.

The reference circuit we proposed refers to the threshold voltage of a MOSFET. Therefore, the absolute value of the output voltage changes significantly with process variation. In other words, the circuit can monitor a process condition of the
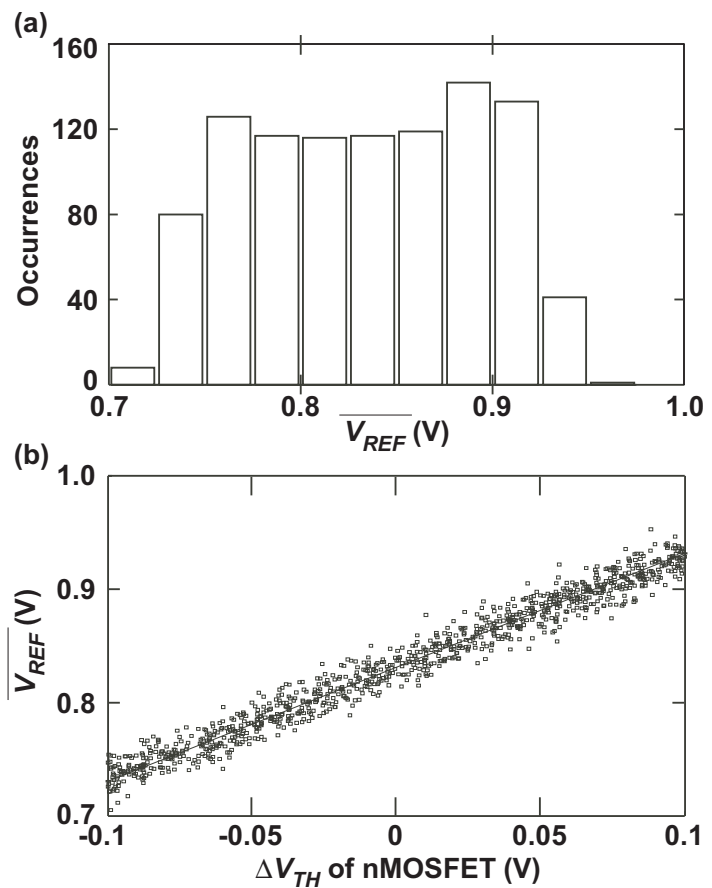

Fig. 3. Simulated results of the output voltage from 1 k-point Monte Carlo simulations. (a) Histogram and (b) threshold voltage dependence of the average output voltage.

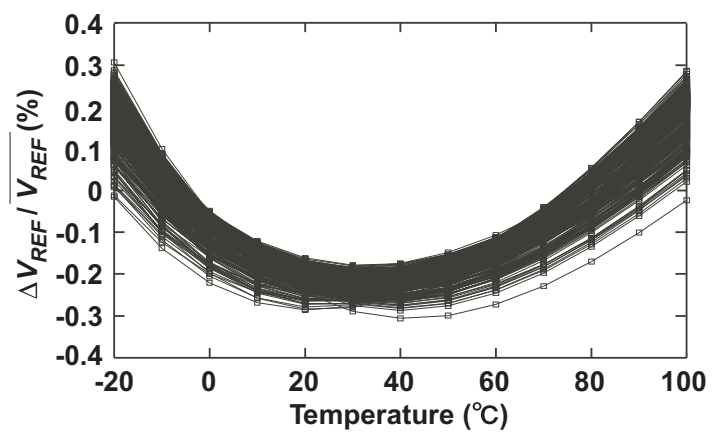

Fig. 4. The errors of the output voltage with temperature derived from Monte Carlo simulations.

TABLE I

PERFORMANCE SUMMARY

\begin{tabular}{c|c}
\hline Process & $0.35-\mu \mathrm{m}, 2$-poly, 4-metal CMOS \\
Temperature range & $-20-100{ }^{\circ} \mathrm{C}$ \\
$V_{D D}$ & $1.5 \mathrm{~V}$ \\
$V_{R E F}$ & $830 \mathrm{mV}(\mathrm{TYP})$. \\
Power & $1.2 \mu \mathrm{W}\left(T=100{ }^{\circ} \mathrm{C}\right)$ \\
$\Delta V_{R E F} / \overline{V_{R E F}}$ & $\pm 0.3 \%\left(T=-20-100{ }^{\circ} \mathrm{C}\right)$ \\
& $\pm 0.5 \%\left(V_{D D}=1.2-3 \mathrm{~V}\right)$ \\
\hline
\end{tabular}

chip. As an example of the application, the reference voltage can be used as a part of the performance compensation system by monitoring its output voltage, which changes with process condition, not only in subthreshold LSIs, but also in standard CMOS LSIs.

\section{REFERENCES}

[1] K. Ueno, T. Hirose, T. Asai, and Y. Amemiya, "CMOS smart sensor for monitoring the quality of perishables," IEEE Journal of Solid-State Circuits, vol. 42, no, 4, pp. 798-803, 2007.

[2] Y. Taur, T.H. Ning, "Fundamentals of Modern VLSI Devices," Cambridge University Press, 2002. 